\title{
Deshalb Kultur!
}

Vermutlich kennt sie jeder von Ihnen, die Frage: Warum Kultur? Während der Corona-Krise haben wir viel Zuspruch erfahren, als wir weiterhin Bücher verliehen und den Lesesaal wieder (eingeschränkt) geöffnet haben. Es wurde aber auch vielfach kritisiert, das sei nicht lebensnotwendig oder systemrelevant. Angesichts des hohen Finanzierungsbedarfs zur Bewältigung der Krise werden in den nächsten Monaten die Stimmen laut, die verlangen, insbesondere bei der Kultur zu sparen. Stuttgart und der Südwesten sind aber wesentlich von einem attraktiven Kulturangebot geprägt und profitieren vielfältig davon. Ich möchte Sie deshalb bitten, die folgenden Argumente zu prüfen und soweit Sie diese teilen, sie auch weiter zu kommunizieren. Vielleicht erinnern Sie sich konkreter Momente, wo Ihnen kulturelle Zeugnisse geholfen haben. Sprechen Sie darüber in Ihrem Umfeld, versuchen Sie, hierzu die Entscheidungsträger zu erreichen. Die Kultur - und unsere Landesbibliothek - brauchen Sie als Resonanzkörper auch angesichts der anstehenden Wahlen. Kultur und Wissen kommen nicht "aus der Steckdose", sind keineswegs selbstverständlich.

Kultur schafft Nähe. Viele Menschen erfahren das erforderliche Distanzhalten als sehr belastend. Viele müssen aufgrund von Vorerkrankungen oder Alter sich weitgehend isolieren. In kulturellen Zeugnissen finden viele über Ablenkung und Unterhaltung hinaus emotionale Unterstützung und Trost. In zahlreichen Stücken suchen sie die fruchtbare Auseinandersetzung von Autoren, Komponisten, Interpreten und Künstlern mit grundsätzlichen Fragen menschlicher Existenz und gesellschaftlichen Zusammenlebens. Viele Texte geben Orientierung und Zusammenhangswissen in einer in der engen Abfolge der Nachrichten oft widersprüchlich erscheinenden Situation. Freude, Nähe, Auseinandersetzung und Interaktion mit künstlerischen Werken leiden, wenn nur digitale Surrogate zur Verfügung gestellt werden können.
Künstlerischer Ausdruck ist gelungene Kommunikation. Sie erfolgt durch eine vorweggenommene Einbeziehung des Betrachters, Hörers oder Lesers. Sie ist das Muster von Empathie und respektvoller Kommunikation, auf welche wir insbesondere in Krisenzeiten angewiesen sind. Der Umgang mit Kunst vermittelt Geduld, Aufmerksamkeit, Umsicht, Takt, gutes Argument und gelungenen Ausdruck. Kultur und Bildung fördern die Entfaltung unserer Kreativität unter Berücksichtigung der Kontexte und Situationen. Kritik und Provokation bilden dabei wichtige Mittel gegen Beliebigkeit, Mainstream, Vorurteil und Verflachung. Die Künste sollten nicht aus den Lehrplänen verschwinden.

Kultur bildet gemeinsame Grundlagen. Wie im Sport finden auch in der Kultur viele Menschen über gemeinsame Interessen und Leidenschaften zusammen. Zusammen etwas großartig zu finden, ist der Ausgangspunkt vieler Beziehungen. Auch jenseits von Freundschaften führt dies zur Bildung formeller und informeller Gruppen des Austausches und des gesellschaftlichen Engagements. Ohne diese faszinierenden Gegenstände unseres gemeinsamen Interesses wäre unser Leben ärmer und der gesellschaftliche Zusammenhalt dünner. Ohne diese vielfältigen Gruppen, Initiativen, Vereine und Kirchengemeinden mit ihrem ehrenamtlichen Engagement wären Städte und Gemeinden überfordert, wäre kein Staat zu machen. Kultur schafft lebensnotwendige Freiräume und Netze jenseits der staatlichen Organisation. Die CoronaKrise zeigt erneut, wie notwendig es ist, den Zugang zu Kultur zu erleichtern, das Publikum nicht nur im Bildungsbürgertum zu suchen, auf dass die Eckensee-Randale nicht zum Menetekel wird.

Kultur stärkt. Kreativität und Kultur sind gefragt, wenn es darum geht, den durch die Corona-Krise zutage getretenen Schwächen unserer Gemeinwesen und des internationalen Zusammenhalts zu begegnen. Kultur bildet Brücken. Aus der Auseinandersetzung mit Kultur, durch die Mitwirkung an kulturellen Projekten können Menschen ihr 
gestalterisches Potential erfahren, spüren sie ihre Wirkmächtigkeit. In Kunst und Kultur werden andere Lebensentwürfe sichtbar, ist Wandel erfahrbar, prüfbar und durchspielbar, eröffnen sich Perspektiven, entsteht Neues.

Wissen teilen. Wissenschaftliche Bibliotheken sind der Platz, an dem eine selbstständige, kritische Auseinandersetzung mit dem kulturellen, wissenschaftlichen und künstlerischen Denken und Agieren in großer Breite und historischer Tiefe möglich ist. Wissenschaftliche Aussagen und Diskussionen können hier anhand entsprechender Publikationen geprüft und nachvollzogen werden. Die Vielfalt der methodischen Ansätze, die diskursive Herausbildung der Erkenntnisse und die Grenzen der gegebenen Erklärungen werden in den Publikationen fassbar. Und natürlich ist wissenschaftliches Arbeiten ohne die Berücksichtigung der einschlägigen Publikationen unmöglich.
Kontexte herstellen. Durch die Fülle ihres Materials gelingt es in der Bibliothek, die Bedingungen unserer Gesellschaften, Alltagskulturen, aber eben auch wissenschaftliche, künstlerische, politische Auseinandersetzungen verstehbar zu machen. Die Wahrnehmung dieser Bezüge ist die Grundlage, wenn man nicht in teilmythologische Erklärungsgebilde zurückfallen oder verheerende Simplifizierungen attraktiver erscheinen lassen möchte.

Sicher können Sie noch weitere Gesichtspunkte beisteuern, haben Sie besondere Beispiele. Ich würde mich freuen. Wir werden in den nächsten Monaten verstärkt den Dialog suchen, um den Resonanzraum der Kultur für die politischen Entscheidungsträger besser wahrnehmbar zu machen. Wir brauchen Ihr Mittun! Teilen Sie Ihre Erfahrungen und Überzeugungen, sprechen Sie mich an oder schicken Sie mir Ihre Beispiele, Ideen oder Unterstützungsmöglichkeiten!

Rupert Schaab

\section{Hölderlin 2020 in der WLB, Teil 2}

\section{Ausstellung „Aufbrüche - Abbrüche. 250 Jahre Friedrich Hölderlin"}

Der Ausstellungstitel „Aufbrüche - Abbrüche“ wurde gewählt, um zentrale, sich wiederholende Merkmale von Hölderlins Leben, Werk und Rezeption widerzuspiegeln, woran sich die Themen der Ausstellung anknüpfen, die bereits im letzten Heft des WLBforum beschrieben wurden. Wie sich herausstellte, wurde der Titel aber ebenso zum Sinnbild der gesamten Zeit, in der die Jubiläumsausstellung die Arbeit der Bibliothek und speziell des Hölderlin-Archivs begleitet hat - von den komplexen Vorbereitungen der Ausstellung über die sich immer wieder verändernden Rahmenbedingungen bis hin zur Corona-Pandemie. Der hoffnungsvolle Aufbruch in das große Jubiläumsjahr 2020 mündete schließlich in einen radikalen Abbruch der Aktivitäten, bedingt durch die in dieser Form nicht für möglich gehaltene Pandemieentwicklung im Frühjahr 2020 und den plötzlichen Lockdown.

Doch dieser Stillstand währte nur kurz. Es deutete sich schon schnell wieder ein neuer Aufbruch an. Durch die notwendige Verschiebung der Ausstellung eröffnete sich auch die Chance auf eine Präsentation der Ausstellung im neuen Erweiterungsbau, wie es bereits fester Bestandteil der ersten Planungen war. Verbunden damit waren natürlich wieder erneute Umgestaltungen, um die Ausstellung den neuen räumlichen Bedingungen anzupassen. Die Arbeit ging also während der Corona-Krise kontinuierlich weiter. Und durch wiederholte Verzögerungen bei der Inbetriebnahme des Erweiterungsbaus wurden auch die Planungen des Aufbaus im Sommer erschwert, sodass sich die an der Vorbereitung Beteiligten bis zur Eröffnung der Ausstellung stets neuen Herausforderungen gegenübergestellt sahen.

Ganz besonders zu danken ist deshalb den Mitarbeiterinnen des Hölderlin-Archivs, insbesondere Ulrike Seegräber und Angelika Votteler für ihren unermüdlichen Einsatz, ferner der Ausstellungsreferentin, Dr. Ida Bentele, die bis zu ihrem Abschied im Juli 2020 